\title{
1 Evolutionary trajectories of three independent neo-sex chromosomes in
}

\section{Drosophila}

3

4 Masafumi Nozawa ${ }^{1,2^{*}}$, Yohei Minakuchi ${ }^{3}$, Kazuhiro Satomura ${ }^{1, \dagger}$, Shu Kondo ${ }^{4}$, Atsushi Toyoda ${ }^{3}$, and

5 Koichiro Tamura ${ }^{1,2}$

6

$7 \quad{ }^{1}$ Department of Biological Sciences, Tokyo Metropolitan University, Hachioji, Tokyo 192-0397, Japan

$8 \quad{ }^{2}$ Research Center for Genomics and Bioinformatics, Tokyo Metropolitan University, Hachioji, Tokyo

9 192-0397, Japan

$10{ }^{3}$ Comparative Genomics Laboratory, Department of Genomics and Evolutionary Biology, National

11 Institute of Genetics, Mishima, Shizuoka 411-8540, Japan

$12{ }^{4}$ Invertebrate Genetics Laboratory, National Institute of Genetics, Mishima, Shizuoka 411-8540, Japan

13

14 * Corresponding author: Masafumi Nozawa, manozawa@tmu.ac.jp

$15 \dagger$ Present address: Department of Information Science and Technology, Hokkaido University, Sapporo,

16 Hokkaido 060-0814, Japan

17 Running title: Evolution of sex chromosomes in Drosophila

18 Keywords: dosage compensation, gene regulation, pseudogenization, expression pattern 


\section{ABSTRACT}

Dosage compensation (DC) on the $\mathrm{X}$ chromosome is a mechanism to counteract the deleterious

22 effects by gene loss from the Y chromosome. However, DC cannot work efficiently if the X chromosome

23 also degenerates. This indeed occurs in the neo-sex chromosomes in Drosophila miranda, where neo-X as

24 well as neo-Y chromosomes are under accelerated pseudogenization. To examine the generality of this

25 pattern, we investigated the evolution of two additional neo-sex chromosomes that independently

26 emerged in $D$. albomicans and $D$. americana and compared their evolutionary processes with that in $D$.

27 miranda. Comparative genomic and transcriptomic analyses revealed that the pseudogenization rate on neo-X is also accelerated in the two species (though lesser extent in D. americana). We also found that neo-X-linked genes whose neo-Y homologs are pseudogenized tend to be upregulated more stringently than those whose neo-Y homologs remain functional. Moreover, the genes under strong functional constraints and highly expressed in the testis tended to remain functional on neo- $\mathrm{X}$ and neo-Y,

32 respectively. Focusing on the $D$. miranda and $D$. albomicans neo-sex chromosomes that independently

33 emerged from the same autosome, we further found that the same genes tend to have been pseudogenized

34 in parallel on neo-Y. Those genes include $I d g f 6$ and $J h I-26$ whose functions seem to be unnecessary or

35 could be even harmful for males. These results indicate that neo-sex chromosomes in Drosophila share a

37 chromosomes in a variety of organisms to avoid being an evolutionary dead-end.

$38(247 / \max 250$ words $)$ 


\section{INTRODUCTION}

Sex chromosomes are widely present in divergent groups of organisms (Bachtrog et al. 2014).

41 Species with sex chromosomes can maintain a stable sex ratio (i.e., 1), irrespective of their surrounding environment, which may be advantageous under certain conditions. However, once sex chromosomes emerge from a pair of autosomes by acquiring sex-determining and sexually antagonistic genes, the proto$\mathrm{X}$ and proto-Y chromosomes mostly stop meiotic recombination to retain stable sex determination, resulting in Y chromosome (hereafter, Y) degeneration (Charlesworth et al. 2005). Consequently, the number of genes on $\mathrm{Y}$ is much smaller than that on the $\mathrm{X}$ chromosome (hereafter, $\mathrm{X}$ ) in many species

47 (Koerich et al. 2008; Cortez et al. 2014; Zhou et al. 2014; Dupim et al. 2018; Bracewell and Bachtrog 2020). Therefore, the number of many X-linked genes become imbalanced, i.e., two in females and one in males. Thus, Y degeneration could potentially be deleterious for males and eventually the entire species with sex chromosomes.

Dosage compensation (DC) as a mechanism to counteract this dosage imbalance of X-linked genes between sexes was originally proposed by Muller (1932) and later more clearly by Ohno (1967) (see Gartler 2014). Many researchers have examined DC since then and reported the presence of global or chromosome-wide DC in many organisms (Disteche 2012; Graves 2016) (see also Ellegren et al. 2007; Zha et al. 2009; Vicoso and Bachtrog 2011 for ineffective DC in some organisms). In Drosophila, the socalled male-specific lethal (MSL) binds to the chromatin entry sites (CES) and triggers lysine acetylation

57 at the 16th residue in histone $\mathrm{H} 4$ (H4K16ac), which in turn stimulates entire male-X upregulation (i.e., 58 global DC) (Alekseyenko et al. 2012; McElroy et al. 2014; Valsecchi et al. 2021). This global DC seems

59 to be also present in the young sex chromosomes, neo-sex chromosomes (hereafter, neo-X and neo-Y, 


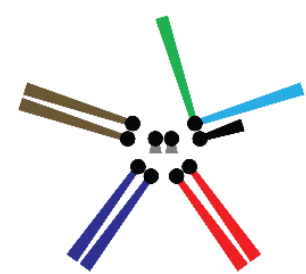

Ancestor

(D. pseudoobscura)

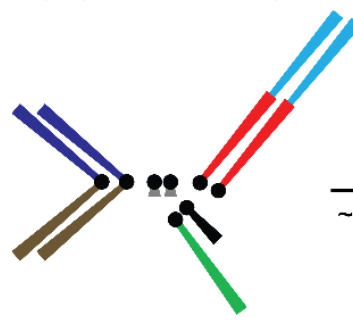

Ancestor

(D. nasuta)

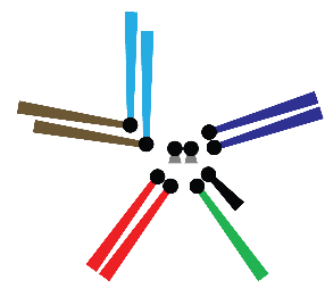

Ancestor

(D. texana)

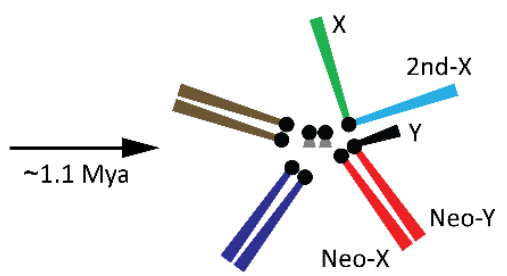

D. miranda

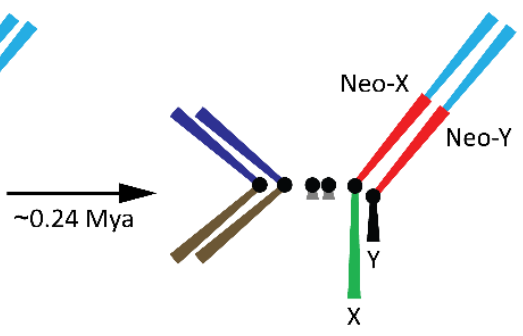

D. albomicans

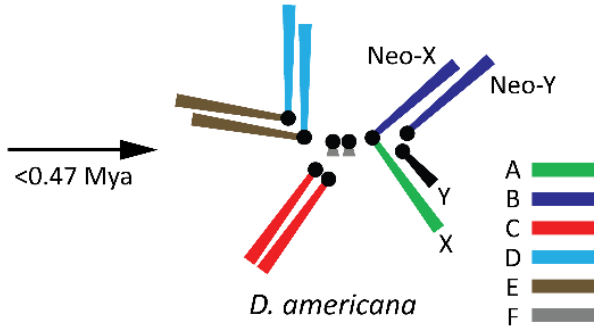

64 Fig. 1: Karyotypes of the three Drosophila species and their ancestors. Male karyotypes are shown. Each chromosome color corresponds to the color of Muller elements shown on the bottom-right corner.

66

67

68

69

70

71

72

73

74

75

In addition to global DC, Nozawa et al. (2018) reported that individual neo-X-linked genes may be upregulated in response to pseudogenization of neo-Y-linked homologs in D. miranda [i.e., gene-bygene or localized DC; see also Nozawa et al. (2014)]. The presence of gene-by-gene DC seems particularly important at the initial stage of sex chromosome evolution when global DC has not yet been established, to counteract the deleterious effects caused by the sequential losses of individual neo-Ylinked genes. However, the pseudogenization rate is accelerated on not only neo-Y, but also neo-X in $D$. miranda (Nozawa et al. 2016), implying that acquiring sex chromosomes may potentially be more deleterious than previously recognized. Yet, whether the observations in D. miranda are true for other species and there is a common evolutionary trajectory of sex chromosomes remains unexplored. To 
address this question, we need to investigate additional young sex chromosomes with independent

77 origins.

In this study, we therefore investigated two other neo-sex chromosomes that originated independently in the Drosophila lineages. One such neo-sex chromosome emerged in D. albomicans 0.24 million years ago (Mya) after the divergence from D. nasuta (Satomura and Tamura 2016; Wei and Bachtrog 2019; Mai et al. 2020). The neo-sex chromosome in this species emerged via the fusion of each chromosome 3 (Muller elements C and D) to the canonical X and Y, respectively (Fig. 1). The other neosex chromosome focused on in this study is present in D. americana. The neo-sex chromosome in this species is likely to have emerged $<0.47$ Mya after splitting from its sibling species, D. texana (Vieira et al. 2003). One of the chromosome 4 (Muller element B) was fused to $X$ and became neo-X, and consequently, the other unfused chromosome 4 became neo-Y (Fig. 1). We also re-investigated the neosex chromosome (Muller element C) in D. miranda, because its genome sequence has greatly been updated (Mahajan et al. 2018; Bachtrog et al. 2019) after the previous studies (Nozawa et al. 2016; 2018).

Analyzing the neo-sex chromosomes in the three species and the orthologous autosomes in their closely-related species, we here report the evolutionary trajectories of the three independent neo-sex on neo-X are shared among the three Drosophila species. It should be mentioned that Muller element C became the neo-sex chromosomes independently in $D$. miranda and D. albomicans (Fig. 1). Since genes on the same Muller element are largely orthologous, we also addressed whether parallel evolution occurred at the gene level in these two neo-sex chromosomes.

\section{$97 \quad$ RESULTS}

\section{Genomes and transcriptomes of the nine Drosophila species} combination with a short-read sequencer (HiSeq 2500; Illumina, San Diego), we sequenced and assembled the genomes of D. albomicans, D. nasuta, D. kohkoa, D. americana, D. texana, and D. 
novamexicana (Table S1). The PacBio RS II-based genome sequence of D. albomicans (strain no. 15112-

METHODS for details).

For D. albomicans and D. nasuta, the contig N50 is $\sim 22.0 \mathrm{Mb}$ and $\sim 17.9 \mathrm{Mb}$, respectively (Table identified as complete open reading frames (ORFs) (Table S3).

118 bodies of females and males at the larval, pupal, and adult stages (Table S4). The number of functional

119 protein-coding genes ranged from $\sim 9,700$ to $\sim 13,900$ depending on the species (Table S5). To estimate

120 the expression level of each gene, we also conducted RNA-seq in the ovary and testis (Table S4). The

121 chromosomal location, expression level, and functionality of each gene and transcript are listed in Tables

122 S6 and S7, respectively.

\section{Proportion of pseudogenes on neo-sex chromosomes}

Bachtrog et al. (2019) reported that the number of genes on Y/neo-Y significantly increased after

126 becoming neo-sex chromosomes in D. miranda. We also confirmed that the number of genes on Y/neo-Y 127 was $\sim 3.4$ times greater than that on neo-X (Fig. 2A). However, $\sim 80 \%$ of the genes were classified as 
pseudogenes due to ORF disruption and/or cessation of transcription. The number of functional genes was each category (i.e., functional, silenced, disrupted, silenced-disrupted, and unclassified genes) were put and 2,031 groups, respectively; Fig. S1). Therefore, although the total number of genes on Y/neo-Y is

133 significantly greater than that on neo-X, Y/neo-Y has considerably degenerated in $D$. miranda and the 134 functional repertoire on $\mathrm{Y} / \mathrm{neo}-\mathrm{Y}$ is smaller than that on neo-X.
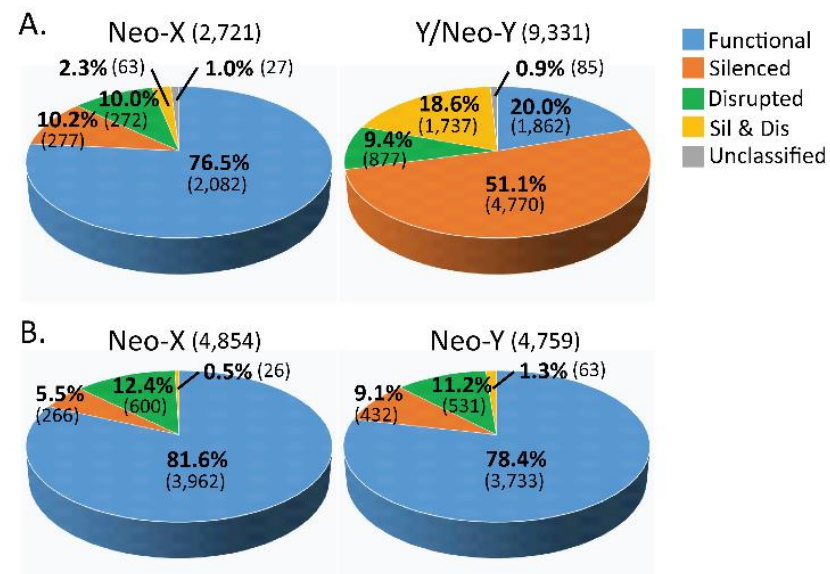

C. $\quad \mathrm{Neo}-\mathrm{X}(2,238)$
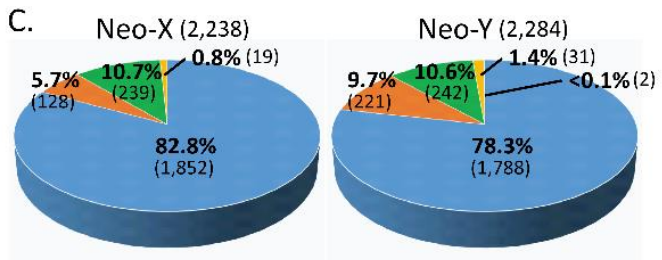

Fig. 2: Classification of neo-X- and neo-Y-linked genes in (A) Drosophila miranda, (B) D. albomicans, and silenced-and-disrupted genes are pseudogenes. 
144 of orthologous gene groups was similar to the number of genes (Fig. S1), indicating that gene duplication

145 was not common on the neo-sex chromosomes after their emergence in these two species. Therefore,

146 degeneration and specialization of neo-sex chromosomes have less proceeded in these two species

147 compared with those in D. miranda (see also Wei and Bachtrog 2019).

149 Gene-by-gene and global DC on neo-Xs

Using the $R_{\text {Lin }}$ index (Lin et al. 2012), Nozawa et al. (2018) reported gene-by-gene DC on the $D$.

151 miranda neo-X. Specifically, the DC level (i.e., the $R_{\text {Lin }}$ value) for the neo-X-linked genes with

152 pseudogenized neo-Y-linked homologs $\left(\mathrm{X}_{\mathrm{F}}-\mathrm{Y}_{\mathrm{P}}\right)$ was significantly greater than that for the neo-X-linked

153 genes with functional neo- $\mathrm{Y}$-linked homologs $\left(\mathrm{X}_{\mathrm{F}}-\mathrm{Y}_{\mathrm{F}}\right)$. The updated datasets confirmed this pattern in all

154 tissues examined in D. miranda (Fig. 3A). In D. albomicans, the trend was the same as that in $D$.

155 miranda, but the difference in the $R_{\mathrm{Lin}}$ value between the $\mathrm{X}_{\mathrm{F}}-\mathrm{Y}_{\mathrm{F}}$ and $\mathrm{X}_{\mathrm{F}}-\mathrm{Y}_{\mathrm{P}}$ groups was significant only in

156 the larva and adult, but not in the pupa and testis (Fig. 3B). In D. americana, the difference was

157 significant in the pupa and adult, but not in others (Fig. 3C). Therefore, the gene-by-gene DC seems to be 158 also present on neo-Xs in the two species, but less conspicuous compared with D. miranda. 

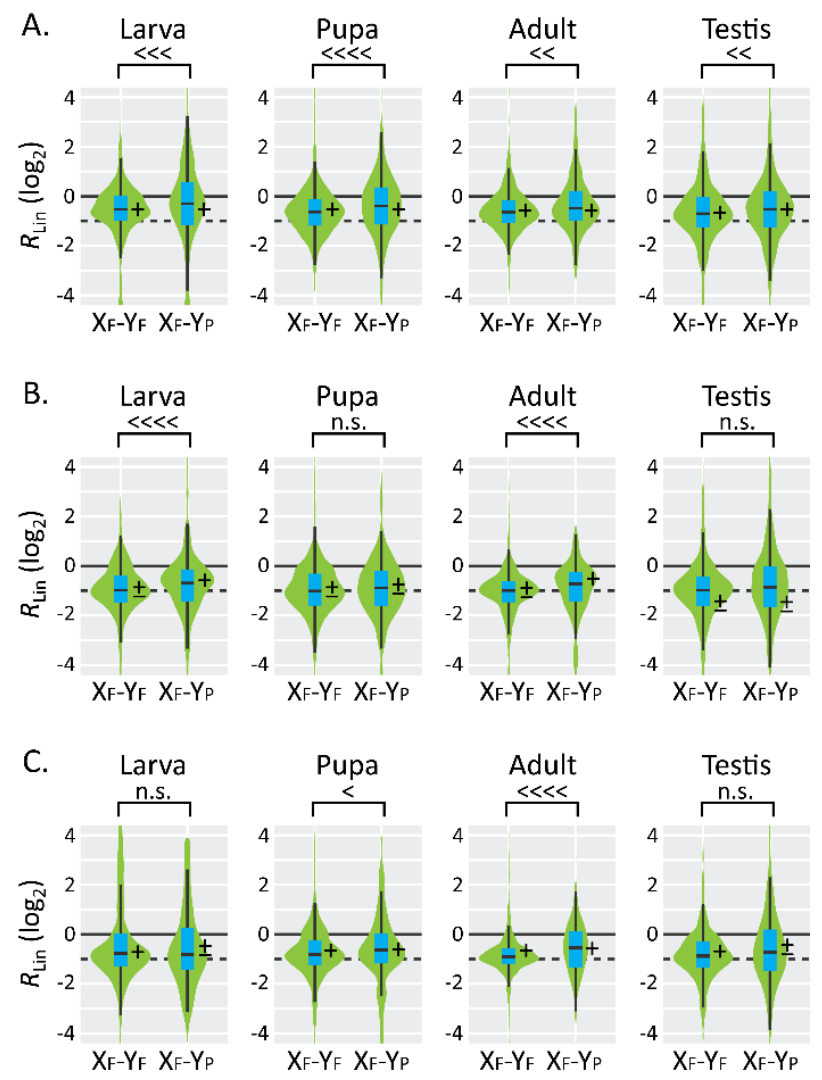

Fig. 3: Relationship between the functionality of neo-Y-linked genes and the DC of neo-X-linked homologs in the larva, pupa, adult, and testis (from left to right) of (A) Drosophila miranda, (B) D. albomicans, and (C) D. americana. For computing $R_{\text {Lin, }}$ the corrected fragments per kilobase of exon per million mapped reads (cFPKM, FPKM normalized by median, see SUPPLEMENTARY METHODS for details) was used. $X_{F}-Y_{F}$, a group of genes with functional neo- $X$-linked and neo- $Y$-linked homologs; $X_{F}-Y_{P}$, a group of genes with functional neo-X-linked homologs and but pseudogenized neo-Y-linked homologs. The numbers of genes analyzed in the $X_{F}-Y_{F}$ and $X_{F}-Y_{P}$ groups are 664 and 435 for D. miranda, 2,449 and 218 for D. albomicans, and 1,204 and 93 for D. americana, respectively. A box plot is also shown on each violin plot. Differences in median between groups were tested based on a permutation test with 10,000 replicates under the null hypothesis of $R_{\mathrm{Lin}}\left(\mathrm{X}_{\mathrm{F}}-\mathrm{Y}_{\mathrm{F}}\right)=R_{\mathrm{Lin}}\left(\mathrm{X}_{\mathrm{F}}-\mathrm{Y}_{\mathrm{P}}\right):<<<<, P<10^{-4} ;<<<, P<10^{-3} ;<<, P<0.01 ;<$,

$171 P<0.05 ;$ n.s., $P \geq 0.05$. A solid line indicates the $R_{\text {Lin }}$ value of $1\left(0 \mathrm{in} \log _{2}\right)$ indicating perfect $\mathrm{DC}$, whereas a

172 broken line corresponds to a value of $0.5\left(-1\right.$ in $\left.\log _{2}\right)$ indicating no DC.,+- , and \pm along each plot means 
that the median $R_{\text {Lin }}$ value is $>0.5,<0.5$, and 0.5 at the $5 \%$ significance level, respectively, based on a bootstrap test with 10,000 replications.

Another difference among the neo-Xs in these species is the $R_{\mathrm{Lin}}$ value for the $\mathrm{X}_{\mathrm{F}}-\mathrm{Y}_{\mathrm{F}}$ group. As already mentioned, the MSL recognizes the CES, which triggers histone acetylation to initiate global DC on the male neo-X in D. miranda (Ellison and Bachtrog 2013). Therefore, in D. miranda, not only $\mathrm{X}_{\mathrm{F}-\mathrm{Y}_{\mathrm{P}}}$ genes, but also $\mathrm{X}_{\mathrm{F}}-\mathrm{Y}_{\mathrm{F}}$ genes showed $R_{\mathrm{Lin}}$ values significantly greater than 0.5 , the expected $R_{\mathrm{Lin}}$ value under no DC (Fig. 3A) (see also Nozawa et al. 2018). In contrast, $\mathrm{X}_{\mathrm{F}-} \mathrm{Y}_{\mathrm{F}}$ gene upregulation was not necessarily conspicuous in $D$. albomicans and $D$. americana. In particular, $R_{\mathrm{Lin}}$ for the $\mathrm{X}_{\mathrm{F}}-\mathrm{Y}_{\mathrm{F}}$ genes was $\sim 0.5$ in all samples examined in D. albomicans (Fig. 3B). Therefore, global DC is unlikely to be established in D. albomicans or D. americana. It should be mentioned that when corrected transcripts per kilobase million mapped reads (cTPM, TPM normalized by the median TPM for each sample; Fig. S2) was used to compute $R_{\text {Lin }}$ instead of cFPKM (Fig. 3) for estimating gene expression levels, the results were qualitatively same.

\section{Accelerated pseudogenization on neo-Xs}

Next, we examined whether the accelerated pseudogenization on neo-X as well as neo-Y reported in D. miranda (Nozawa et al. 2016) occurred on the neo-sex chromosomes in D. albomicans and D. americana. First, we confirmed the accelerated pseudogenization on both neo-X and neo-Y in D. miranda with the updated datasets (Fig. 4A-B). The pseudogenization rate on the neo-X lineage (branch $a$ ) was significantly greater than that on the orthologous autosome in D. pseudoobscura (branch $e)\left(P=1.5 \times 10^{-6}\right.$ by $\chi^{2}$ test). Similarly, the pseudogenization rate on the neo-X lineage (branch $a$ ) was significantly greater than that on the D. miranda ancestral lineage before the emergence of neo-sex chromosome (branch $c$ ) $\left(P=5.5 \times 10^{-10}\right)$. The sum of pseudogenization events on branches $a$ and $c$ was also significantly greater than the sum of those on branches $d$ and $e\left(P=2.9 \times 10^{-3}\right)$. 
A.

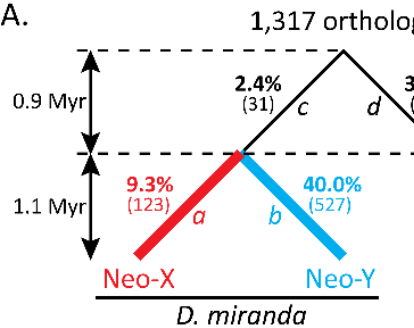

C.

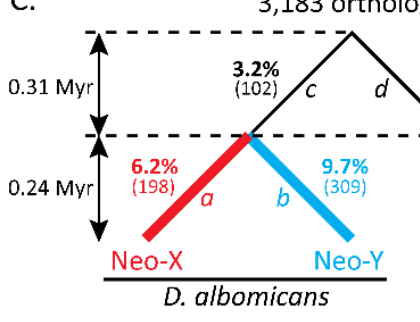

E.

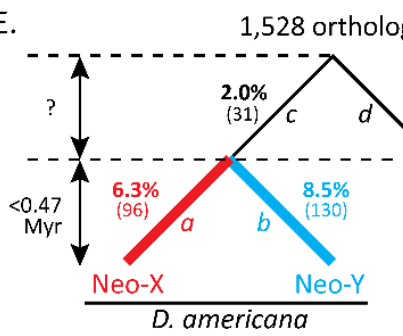

B.

\begin{tabular}{ccc}
\hline Lineage 1 & Lineage 2 & $P$ value \\
\hline$a$ & $b$ & $1.5 \times 10^{-56}$ \\
$a$ & $e$ & $1.5 \times 10^{-6}$ \\
$b$ & $e$ & $7.5 \times 10^{-63}$ \\
$a$ & $c$ & $5.5 \times 10^{-10}$ \\
$b$ & $c$ & $2.9 \times 10^{-78}$ \\
$a+c$ & $d+e$ & $2.9 \times 10^{-3}$ \\
$b+c$ & $d+e$ & $7.0 \times 10^{-69}$ \\
$c$ & $d$ & 0.06 \\
\hline
\end{tabular}

D.

\begin{tabular}{ccc}
\hline Lineage 1 & Lineage 2 & $P$ value \\
\hline$a$ & $b$ & $8.2 \times 10^{-7}$ \\
$a$ & $e$ & $5.6 \times 10^{-9}$ \\
$b$ & $e$ & $1.1 \times 10^{-25}$ \\
$a$ & $c$ & $5.7 \times 10^{-15}$ \\
$b$ & $c$ & $4.8 \times 10^{-38}$ \\
$a+c$ & $d+e$ & $9.0 \times 10^{-4}$ \\
$b+c$ & $d+e$ & $1.2 \times 10^{-13}$ \\
$c$ & $d$ & 0.11 \\
\hline & &
\end{tabular}

F.

\begin{tabular}{ccc}
\hline Lineage 1 & Lineage 2 & P value \\
\hline$a$ & $b$ & $2.4 \times 10^{-2}$ \\
$a$ & $e$ & - \\
$b$ & $e$ & - \\
$a$ & $c$ & - \\
$b$ & $c$ & - \\
$a+c$ & $d+e$ & 0.24 \\
$b+c$ & $d+e$ & $1.6 \times 10^{-3}$ \\
$c$ & $d$ & -
\end{tabular}

Fig. 4: Pseudogenization events before and after neo-sex chromosome emergence. (A, $C$, and $E$ )

Proportions of genes that were pseudogenized in (A) Drosophila miranda, (C) D. albomicans, and (E) D. americana lineages compared with those in their closely-related species. The numbers in parentheses indicate the number of genes pseudogenized in each lineage. The orthologs regarded as functional in the outgroup species (D. obscura, D. kohkoa, and D. novamexicana, respectively) and located on the same Muller element without any inparalogs were used for this analysis. The number of such orthologs is shown above each tree. (B, $D$, and E) Statistical significance in the difference of the pseudogenization rate between lineages of (B) D. miranda, (D) D. albomicans, and (F) D. americana by $\chi^{2}$ test under the null hypothesis of equal pseudogenization rates between lineages. Lineages $a$ to $e$ correspond to the branches in $\mathrm{A}, \mathrm{C}$, and $\mathrm{E}$. 
all statistically significant. In contrast, the difference was not significant in the D. americana lineage (96

$214+31=127$ for $a+c$ and 109 for $d+e, P=0.24$ ), although the trend was qualitatively same (Fig. 4E-F).

215 The difference in the pseudogenization rates on neo-X and neo-Y was also less clear $(P=0.024)$, probably

216 reflecting their recent origin. It should be noted that the pseudogenization rate was not significantly

217 different for most of other chromosomes between the species with neo-sex chromosomes and their close

218 relatives without neo-sex chromosomes (Fig. S3). Therefore, the mutation rate as well as general

219 functional constraints between the former and the latter species would be essentially the same. These

220 results indicate that the three species with neo-sex chromosomes share an evolutionary trajectory with

221 respect to the accelerated pseudogenization on neo-X, although the extent varies with species, possibly

222 due to the age difference among the neo-sex chromosomes.

\section{Pseudogenization pattern on neo-sex chromosomes}

We next examined pseudogenization pattern on neo-X and neo-Y. First, to test whether

pseudogenes on the neo-sex chromosomes were under less functional constraints before being linked to neo-sex chromosomes, we examined the relationship between functionality of neo-sex-linked genes and ratio of nonsynonymous to synonymous nucleotide divergence per site $\left(d_{\mathrm{N}} / d_{\mathrm{S}}\right)$ (Fig. 5). In the $D$. miranda

229 lineage, pseudogenes on neo-X (i.e., $\mathrm{X}_{\mathrm{P}-} \mathrm{Y}_{\mathrm{F}}$ and $\mathrm{X}_{\mathrm{P}}-\mathrm{Y}_{\mathrm{P}}$ genes) tended to have less functional constraints

230 when they were functional in the ancestral lineage before the emergence of neo-X and neo-Y (branch $c$ in

231 Fig. 5A). A similar but less clear tendency was also observed in the D. albomicans and D. americana

232 lineages (branch $c$ in Fig. 5B-C). $X_{P}-Y_{F}$ genes tend to have evolved faster than $X_{F}-Y_{F}$ and $X_{F}-Y_{P}$ on the

233 neo-X lineages in all three species as expected (branch $a$ in Fig. 5A-C). However, $X_{\mathrm{P}}-Y_{\mathrm{F}}$ genes tend to

234 have evolved even faster than $\mathrm{X}_{\mathrm{P}}-\mathrm{Y}_{\mathrm{P}}$ genes, particularly in the D. miranda lineage (middle panel in Fig.

$2355 \mathrm{~A}) . \mathrm{X}_{\mathrm{F}}-\mathrm{Y}_{\mathrm{P}}$ genes, in contrast, did not necessarily evolve faster than other gene categories in the ancestral

236 lineage (branch $c$ ), although they have evolved faster on the neo-Y lineages (branch $b$ ) as expected (Fig.

$2375 \mathrm{~A}-\mathrm{C})$. Therefore, neo-X-specific pseudogenization events have primarily occurred because these genes

238 were under less functional constraints before becoming neo-sex-linked, whereas neo-Y-specific 

much.

241
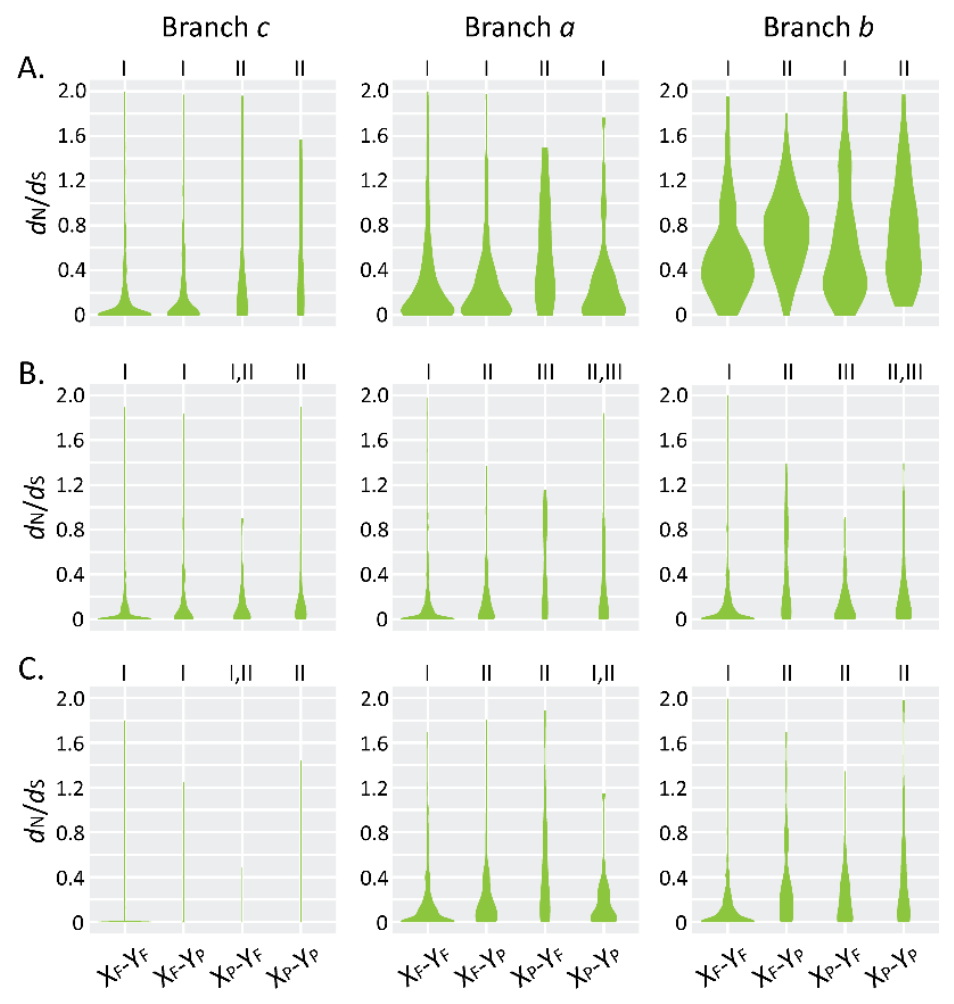

244 synonymous nucleotide divergence per site $\left(d_{N} / d_{s}\right)$ in (A) Drosophila miranda, (B) D. albomicans, and (C)

245 D. americana lineages. Branches $c$ (left), $a$ (center), and $b$ (right) corresponds to those in Fig. 4. Violin

246 plots were used to show $d_{N} / d_{\mathrm{s}}$ distribution. The orthologs regarded to be functional in the outgroup 247 species (D. obscura, D. kohkoa, and D. novamexicana, respectively) and located on the same Muller 248 element without any inparalogs were used for this analysis. The number of genes analyzed for each category is as follows: 681 genes for $X_{F}-Y_{F}, 474$ genes for $X_{F}-Y_{P}, 70$ genes for $X_{P}-Y_{F}$, and 92 genes for $X_{P}-Y_{P}$

250 in the D. miranda lineage; 2,551 genes for $X_{F}-Y_{F}, 277$ genes for $X_{F}-Y_{P}, 166$ genes for $X_{P}-Y_{F}$, and 189 genes

251 for $X_{P}-Y_{P}$ in the $D$. albomicans lineage; 1,267 genes for $X_{F}-Y_{F}, 119$ genes for $X_{F}-Y_{P}, 85$ genes for $X_{P}-Y_{F}$, and

25257 genes for $X_{P}-Y_{P}$ in the $D$. americana lineage. Differences in median values between categories were

253 tested based on a permutation test with 10,000 replicates under the null hypothesis of the equal $d_{N} / d_{\mathrm{S}}$ 
ratio between categories. Same roman numerals indicate $P \geq 0.05$, whereas different numerals mean

Next, we conducted gene ontology (GO) analysis to extract features in functional genes or pseudogenes on neo-sex chromosomes. The analysis detected many GO terms enriched in functional

259 genes and pseudogenes on neo-X and neo-Y (Tables S8-19). However, the trend was largely shared between neo-X and neo-Y of the three species. For example, genes involved in metabolic processes tended to be functional on both neo-X and neo-Y of the three species. On the contrary, genes related to detection of chemical stimuli commonly tended to be nonfunctional on the three neo-sex chromosomes. The shared feature between neo-X and neo-Y in each species may be expected, because they had shared evolutionary history until recently. However, this explanation would be insufficient for the enriched GO terms shared among species. We speculate that the above patterns seem to be general features applicable to all chromosomes. Indeed, chemosensory receptor genes involved in detecting chemical stimuli are 267 known to have been under dynamic turn-over and pseudogenization in many animals (Niimura and Nei 2007; Nozawa and Nei 2007; Nei et al. 2008; Hayakawa et al. 2014).

Therefore, we next examined whether pseudogenization events are associated with spatiotemporal gene expression patterns. For this analysis, genes were classified based on the tissue where

271 the genes were expressed the highest among the samples examined. Ideally, the gene expression level in

272 the ancestral species without neo-sex chromosomes should be used to classify the genes, but obtaining

273 RNA from ancestral species is impossible. We therefore used the gene expression level in the closely-

274 related species without neo-sex chromosomes as a proxy for the gene expression level in the ancestor

275 (i.e., D. pseudoobscura, D. nasuta, and D. texana for D. miranda, D. albomicans, and D. americana, 276 respectively).

277 On neo-X, the proportion of pseudogene was not significantly different among groups in all three

278 lineages (second panel from the right in Fig. 6A-C). On canonical X, the genes expressed the highest in 279 the ovary showed a consistent trend of maintaining their functions, although the trend was statistically 
280 significant only in D. miranda (second panel from the left in Fig. 6A-C). A similar but non-significant

281 pattern was also observed in the D. miranda and D. americana neo-Xs [Fig. 6A, C, see also Nozawa et al.

282 (2016)]. Therefore, neo-X may be in a transitional state toward canonical $\mathrm{X}$ with respect to gene content.

283 On neo-Y, the genes expressed the highest in the testis showed a significantly lower proportion of

284 pseudogenes than those expressed the highest in somatic tissues for all three lineages (rightmost panel in

285 Fig. 6A-C), consistent with previous studies in D. miranda (Kaiser et al. 2011; Nozawa et al. 2018). The

286 genes expressed the highest in the ovary also tended to maintain their functions even after being neo-sex-

287 linked in all three lineages, which was also consistent with the finding in D. miranda (Nozawa et al.

288 2018). It should be mentioned that there was no clear-cut difference among tissues on autosomes

289 (leftmost panel in Fig. 6A-C), probably because of biparental inheritance with equal contribution from

290 males and females. Notably, the general pattern remained unchanged even when TPM rather than cFPKM

291 was used to estimate the gene expression level (Fig. S4). Therefore, pseudogenization pattern shared on

292 neo-Y in the three species would be primarily due to the linkage of the genes to Y. 

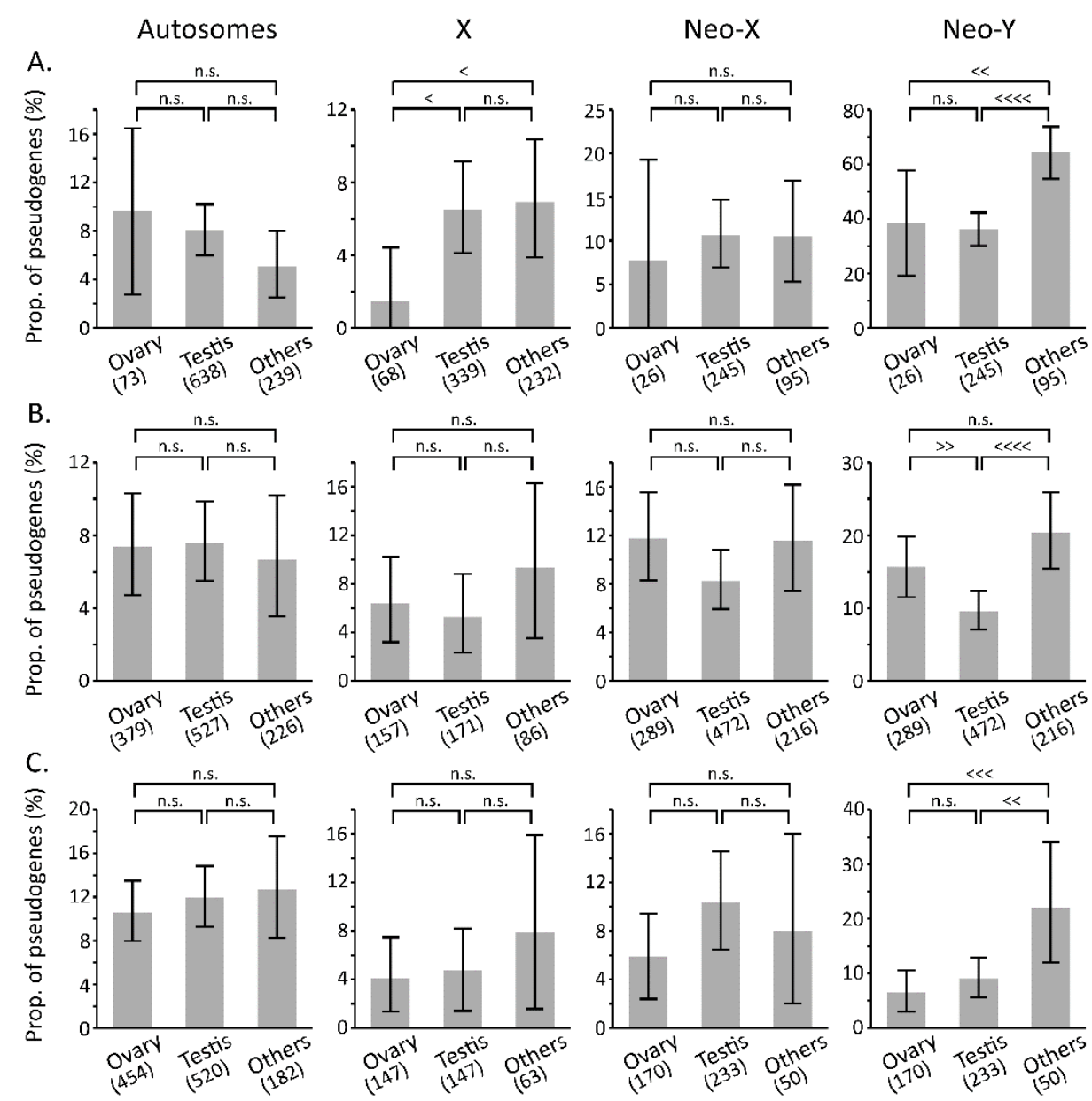

Tissue with maximum expression

Fig. 6: Relationship between spatiotemporal gene expression pattern and pseudogenization. All genes

were classified into three groups based on the tissue with the highest expression in (A) Drosophila

pseudoobscura, (B) D. nasuta, and (C) D. texana, the closely-related species of $D$. miranda, $D$.

albomicans, and D. americana, respectively, based on the cFPKM value. However, to remove the genes

with similar cFPKMs in multiple tissues, only genes with at least two-fold cFPKM in a tissue compared

with that in the other tissues examined were used for this analysis. The orthologs regarded to be

functional in the close relatives (D. pseudoobscura and D. obscura for D. miranda, D. nasuta and $D$. 
bootstrap resampling with 10,000 replicates. Statistical significance between groups was calculated by a permutation test with 10,000 replicates: $<<<<$ or $\gg>>>, P<10^{-4} ;<<<$ or $>>>, P<10^{-3} ;<<$ or $>>$; $P<0.01 ;<$ or $>, P<0.05 ;$ n.s., $P \geq 0.05$.

\section{Parallel pseudogenization on the neo-sex chromosomes in D. miranda and D. albomicans}

The above analyses consistently indicate that the three neo-sex chromosomes largely share a

311 common evolutionary trajectory after their independent emergence. Since Muller element C

312 independently became the neo-sex chromosomes in D. miranda and D. albomicans, we directly examined

313 whether the same orthologs tend to have been parallelly pseudogenized in both lineages (Fig. 7). The

314 result showed that three orthologs were parallelly pseudogenized on the neo-X lineages, which was within

315 a range under random pseudogenization on each lineage (Fig. 7A and D). In contrast, there were 35

316 parallel pseudogenization events on the neo-Y lineages, which was significantly greater than the

317 distribution under random pseudogenization (Fig. 7B and E). It should be noted that the same genes tend

318 to have also been pseudogenized on Muller elements A, D, and E, but the trend was much weaker

319 compared with the pattern on the neo-Y lineages of the two species (Fig. S5). It should also be noted that

320 there was no parallel pseudogenization on the Muller element $\mathrm{C}$ in the ancestral lineage before the

321 emergence of the neo-sex chromosomes (Fig. 7C and F). Therefore, parallel pseudogenization on the two

322 neo-Ys is unlikely to be accidental but likely reflects a common evolutionary trajectory regarding

323 pseudogenization pattern on neo-Y. 
A.

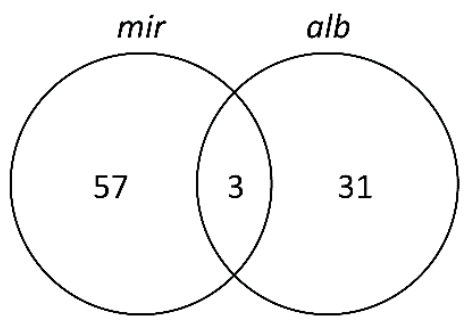

D.

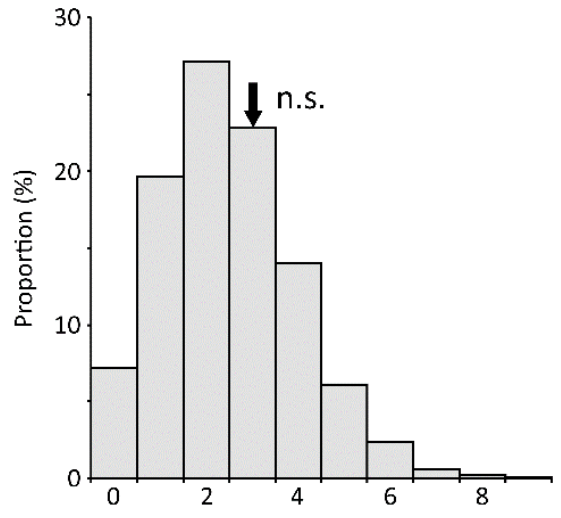

No. of parallel pseudogenization events
B.

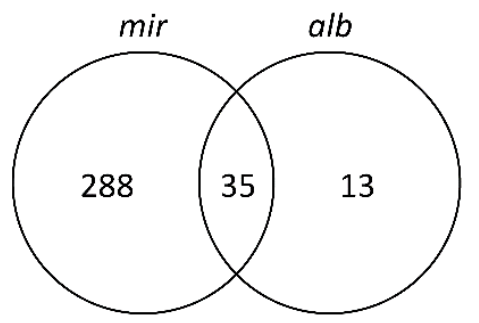

E.

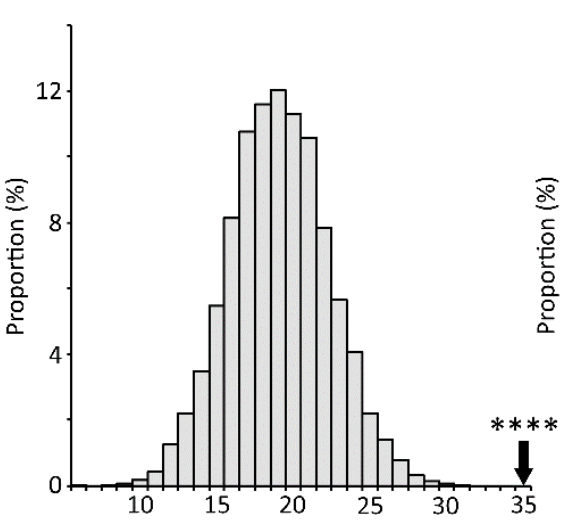

No. of parallel pseudogenization events
C.

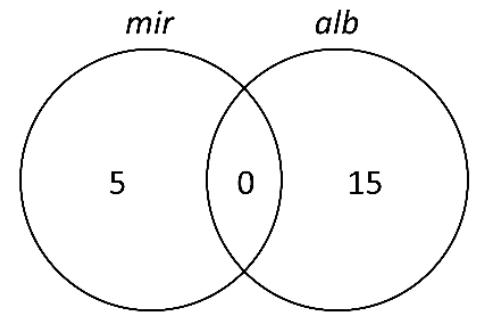

F.

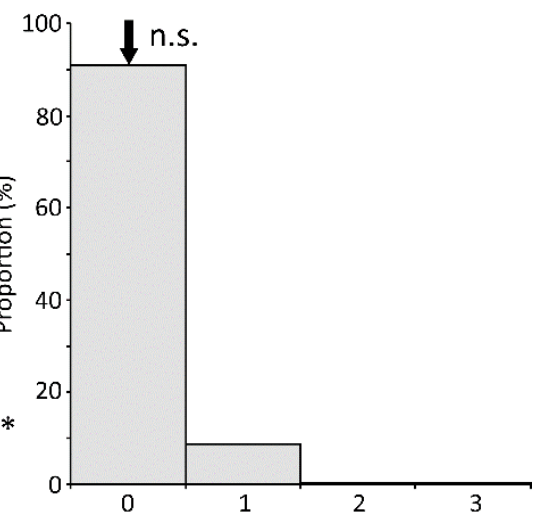

No. of parallel pseudogenization events

Fig. 7: Parallel pseudogenization events on the neo-sex chromosomes in Drosophila miranda (mir) and $D$.

albomicans (alb) lineages. A total of 814 orthologs regarded to be functional in the outgroup species ( $D$.

obscura and D. kohkoa, respectively) and located on the Muller element $C$ without any inparalogs were

used for the analysis. A Venn diagram shows the number of lineage-specific or parallel pseudogenization

Figs. 4A and 4C) of D. miranda and D. albomicans. Distribution of the number of pseudogenization

events based on the permutation test with 10,000 replicates under the assumption of random

pseudogenization on the (D) neo-X, (E) neo-Y, and (F) ancestral lineages (i.e., branches $a, b$, and $c$,

respectively, in Figs. $4 \mathrm{~A}$ and $4 C$ ) of $D$. miranda and D. albomicans is shown below each Venn diagram. An

arrow represents the observed number of parallel pseudogenization events with the following statistical

significance: $* * * *, P<10^{-4}$; n.s., $P \geq 0.05$.

We further searched for unique features of the genes that experienced parallel pseudogenization.

339 The $d_{\mathrm{N}} / d_{\mathrm{S}}$ ratios for the genes that experienced parallel pseudogenization and lineage-specific

340 pseudogenes were similar (Fig. S6), although the ratios for the pseudogenes tended to be higher than 
341 those for functional genes on the neo-Y lineages (branch $b$ in Fig. S6). However, the orthologs of the neo-

342 Y pseudogenes in the closely-related species (D. pseudoobscura for D. miranda and D. nasuta for D.

343 albomicans, respectively) showed tissue-specific expression as indicated by $\tau$ (Yanai et al. 2005) at a

344 significantly greater extent than those of functional genes, although there was no significant difference in

$345 \tau$ between orthologs of lineage-specific and shared pseudogenes (Fig. 8A). The number of expressed

346 tissues also tended to be smaller in the orthologs of shared pseudogenes than those of functional genes

347 and lineage-specific pseudogenes on neo-Y (Fig. 8B). Moreover, the orthologs of shared neo-Y-linked

348 pseudogenes in D. pseudoobscura, closely related to D. miranda, tended to show female-biased

349 expression compared with the orthologs of functional genes and lineage-specific pseudogenes at the larval

350 and pupal stages in D. miranda (Fig. 8C), although the pattern was less clear in the D. albomicans lineage

351 (Fig. 8D). Therefore, the female-biased genes expressed only in a small number of tissues tend to be

352 parallelly pseudogenized on neo-Y. 

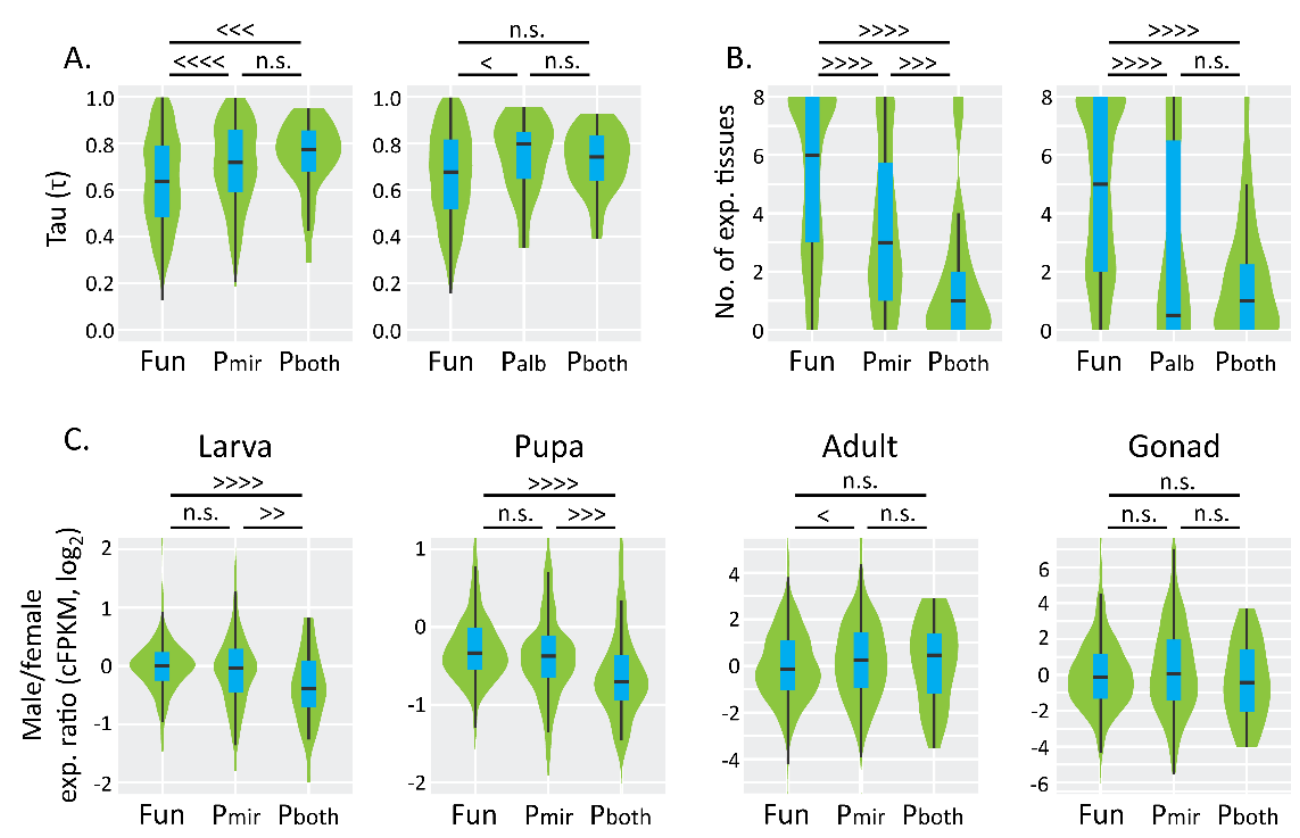

D.
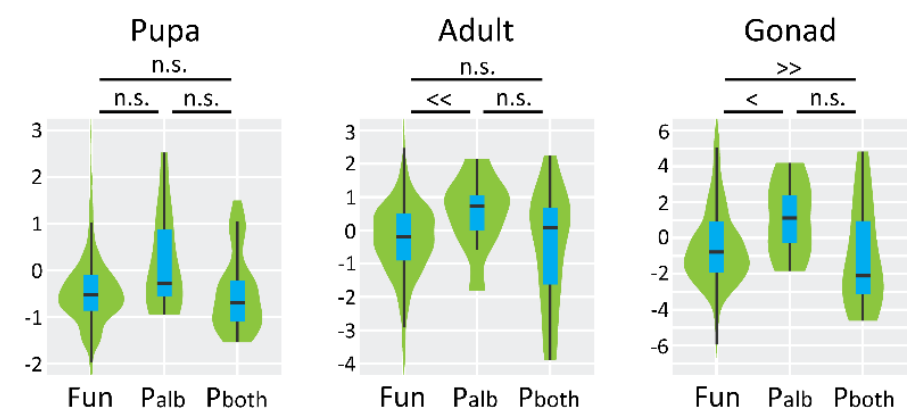

Fig. 8: Relationship between parallel pseudogenization on neo-Y and their expression pattern. (A)

Relationship between pseudogenization pattern and tissue specificity in gene expression $(\tau)$. $\tau$ was computed for orthologs in D. pseudoobscura (left panel) and D. nasuta (right panel), the closely-related species of $D$. miranda and D. albomicans, respectively, as proxies of ancestral species. (B) Relationship between pseudogenization pattern and the number of expressed tissues for each gene. A gene was regarded to be expressed in a tissue if the cFPKM was $\geq 1$ in $D$. pseudoobscura (left panel) and $D$. nasuta (right panel). (C, D) Relationship between pseudogenization pattern and male to female gene expression ratio based on the cFPKM value. Fun, neo-Y-linked genes functional at least in $D$. miranda (left panel in $\mathrm{A}$ 362 and B and all panels in C) or D. albomicans (right panel in A and B and all panels in D); $P_{\text {mir, }}$, neo-Y-linked genes nonfunctional in D. miranda but functional in D. albomicans; $\mathrm{P}_{\mathrm{alb}}$, neo-Y-linked genes 
nonfunctional in D. albomicans but functional in D. miranda; $\mathrm{P}_{\text {both, }}$ neo-Y-linked genes nonfunctional in both $D$. miranda and $D$. albomicans. Differences in median between categories were statistically evaluated based on a permutation test with 10,000 replicates: $\ll<<<$ or $\gg \gg \gg, P<10^{-4} ;<<<$ or $\gg \gg, P<10^{-3}$; $<$ or $>>$; $P<0.01 ;<$ or $>, P<0.05 ;$ n.s., $P \geq 0.05$.

\section{DISCUSSION}

A variety of sex-determination systems have come into existence in a long history of life. Among

371 them, sex chromosomes have particularly been widespread and repeatedly emerged in many lineages.

372 However, if many gene losses from Y are considered, sex chromosome acquisition is not a simple

373 evolutionary process. In this study, we investigated if there is a common evolutionary trajectory to avoid

374 the neo-sex chromosomes being evolutionary dead-end, using the three Drosophila species that

375 independently acquired neo-sex chromosomes and their closely-related species without neo-sex

376 chromosomes. The shared features that we found in this study can be summarized as follows: 1) The

377 gene-by-gene or localized DC operates on neo-X-linked genes. 2) The pseudogenization rate was

378 accelerated on neo-X. 3) The neo-Y-linked genes with the highest expression in the testis or ovary tend to

379 remain functional. 4) The same orthologs tend to be parallelly pseudogenized on neo-Y in D. miranda and

D. albomicans.

\section{DC on neo-X in Drosophila}

When individual Y-linked genes are pseudogenized, immediate upregulation of X-linked

384 homologs would be critical to counteract the deleterious effects of the pseudogenization. In this study, we

385 found the gene-by-gene upregulation of neo-X-linked homologs in the three species with independent

386 origins of neo-sex chromosomes, which has likely played an important role in diminishing the effect of

387 losing neo-Y genes. However, the gene-by-gene DC was less clear in D. albomicans and D. americana,

388 with younger neo-sex chromosomes, compared with the DC in D. miranda with older neo-sex

389 chromosomes. These results imply that the time to fully develop the gene-by-gene DC mechanism is 
insufficient for the former two species. It should be mentioned that this implication is not necessarily consistent with the previous report that a certain portion of the neo-X-linked genes already have a potential of upregulation (i.e., gene-by-gene DC) before losing their neo-Y homologs in D. miranda (Nozawa et al. 2018). Yet, their analysis is unlikely to be conclusive as they also noticed large sampling errors in estimating the pseudogenization time for each neo-Y-linked gene due to the small sequence divergence between the neo-sex chromosomes in D. miranda. Hence, we did not conduct the same analysis for $D$. albomicans and $D$. americana, in which the sequence divergence between neo-X and neo$\mathrm{Y}$ is even smaller. Therefore, how quickly the mechanism of gene-by-gene DC is established after neo-Ylinked gene loss remains unclear. We are currently making the mutants of D. miranda and D. albomicans in which several functional neo-Y-linked genes are knocked out and see if the neo-X-linked homologs are immediately upregulated in one generation.

We also found that global DC as well as gene-by-gene DC operate on the neo-X in D. miranda as already reported (Ellison and Bachtrog 2013; Nozawa et al. 2018), whereas in D. albomicans and D. americana, global DC is unlikely to exist. This observation strengthens the speculation by a previous study (Nozawa et al. 2018) that gene-by-gene DC is developed first in the early stage of sex chromosome evolution and global DC is developed later and gradually takes over the role of gene-by-gene DC as Y degeneration proceeds. Under this scenario, the neo-Xs in D. albomicans and D. americana are so young

407 that global DC has not been established yet, whereas the neo-X in D. miranda is in a transient stage from 408 gene-by-gene to global DC. Consistent with this scenario, the neo-Xs that independently originated in $D$. 409 melanica and D. robusta about 4-15 Mya (Flores et al. 2008) have already acquired the MSL binding 410 sites, i.e., the global DC mechanism (Ellison and Bachtrog 2019). On the contrary, the neo-X with an age 411 of $\sim 0.85$ Mya in D. busckii is unlikely to acquire the global DC (Zhou and Bachtrog 2015). These 412 observations imply that approximately one million years may be necessary for neo-X to at least partially 413 develop global DC mechanism in Drosophila species. It should be mentioned that neo-Y degeneration is 414 not conspicuous in D. albomicans and D. americana. Therefore, the effects of gene loss from neo-Y on 415 fitness may be limited even without strong gene-by-gene DC. 


\section{Accelerated pseudogenization on neo-X and neo-Y in Drosophila}

As discussed above, gene-by-gene DC certainly operates on neo-X, which is likely critical at the

early stage of sex chromosome evolution for organisms to maintain their fitness. However, gene-by-gene

DC is unlikely to completely mitigate the deleterious effects caused by neo-Y-linked gene loss, because

421 the pseudogenization rate is also accelerated on neo-X, at least in D. miranda and D. albomicans (and the

422 trend is the same in D. americana as well). If a gene is pseudogenized or lost from neo-X, gene-by-gene

423 DC cannot operate on its neo-Y-linked homolog. However, the neo-X-linked pseudogenes tend to have

424 been under less functional constraints when they were functional on an autosome. Thus, the fitness effects

425 of these pseudogenizations seem to be minimized.

What kinds of evolutionary forces have driven this accelerated rate of pseudogenization on neo-

427 sex chromosomes? For neo-Y, suppression of meiotic recombination and small effective population size

428 (one-fourths compared with the size of an autosome) would be sufficient explanations. For neo-X,

429 however, the situation is more complex. The effective population size of neo-X is expected to be three-

430 fourths compared with that of an autosome under equal sex ratio. However, unlike many other organisms,

431 most Drosophila species do not recombine at male meiosis (John et al. 2016). Therefore, the proportion

432 of chromosomes which recombine at meiosis is two-thirds for neo-X, greater than two-fourths for an

433 autosome (Charlesworth 2012). Therefore, the inclusive efficacy of natural selection on neo-X and an

434 autosome is expected to be similar (i.e., $3 / 4 \times 2 / 3=1 / 2$ for neo-X and $4 / 4 \times 2 / 4=1 / 2$ for an autosome).

435 Therefore, other forces such as sexual conflict may have significant effects on the fates of genes on neo-

436 sex chromosomes. Indeed, genes likely involved in sexual conflict are reported to show a biased pattern

437 of pseudogenization on the neo-X in D. miranda, which may have played a certain role in reducing the

438 sexual conflict between males and females due to the acquisition of neo-sex chromosomes (Nozawa et al.

439 2016; but see also Bachtrog et al. 2019 for neo-sex chromosomes as a new battleground for sexual

440 conflict). 
The duration of accelerated pseudogenization on neo-X after neo-sex chromosome emergence

would also be an intriguing point to be clarified. In the dipteran lineage, dozens of sex chromosome turnover have occurred (Vicoso and Bachtrog 2015). Because most Y-linked genes in dipteran species, and probably many other species, are pseudogenized, $\mathrm{X}$, but not $\mathrm{Y}$, will be reversed to an autosome (Meisel 2020). Indeed, a large number of genes remain functional on $X$ in many species (Koerich et al. 2008;

Cortez et al. 2014; Zhou et al. 2014; Dupim et al. 2018; Bracewell and Bachtrog 2020). Since chromosome-level assemblies of different ages of $\mathrm{X}$ have recently become available in several Bachtrog 2020), it may be possible to estimate the transition of the pseudogenization rate of $X$ after its emergence in the future.

\section{Pseudogenization pattern on neo-sex chromosomes in Drosophila}

We found that the genes under less functional constraints in the ancestor before neo-sex chromosome emergence are frequently pseudogenized after being neo-X-linked. As already mentioned, this trend would minimize the deleterious effect of acquiring sex chromosomes on the fitness of organisms. In contrast, this trend does not necessarily hold for the neo-Y-linked genes, particularly the genes pseudogenized only on neo-Y. Instead, the spatiotemporal gene expression pattern is likely to be a primary factor in determining the fate of neo-Y-linked genes. Specifically, if a gene is highly expressed in

459 the testis of the closely-related species without neo-sex chromosomes (possibly reflecting the gene 460 expression pattern in the ancestor), it tends to remain functional after being neo-Y-linked. This

461 observation is reasonable because $\mathrm{Y}$ is transmitted only through males, so the genes whose functions are 462 specific or biased to males, such as genes highly expressed in the testis, are likely maintained. 
Indeed, the genes with the highest and second highest expression in the ovary and male tissues, expression in the ovary and other female tissues, respectively, on the D. miranda neo-Y (Nozawa et al. 2018). Although our updated dataset for D. miranda with more stringent criteria to select the genes for the analysis did not reproduce such patterns (the proportions of pseudogenes are 0.43 and 0.37 for the former

472 and the latter, respectively, with $P=0.87$ by permutation test with 10,000 replications), we observed a

473 weak but non-significant pattern in D. albomicans ( 0.14 and 0.17 , respectively, with $P=0.40$ ) and a strong 474 tendency in $D$. americana ( 0.02 and 0.09 , respectively, with $P=0.01)$. Therefore, some genes with the 475 highest expression in the ovary may also be important for males and remain functional on neo-Y.

477 and neo-Y recombine at male meiosis, pseudogenes on neo-Y can theoretically be replaced with neo-X-

478 derived functional genes. Although males do not recombine at meiosis in D. melanogaster and many

479 other Drosophila species as mentioned above (John et al. 2016), D. nasuta, closely related to D.

480 albomicans, recombines during male meiosis (Satomura and Tamura 2016). In addition, neo-X and neo-Y

481 in D. albomicans are likely to have recombined until recently, supported by a large number of shared

482 polymorphisms between neo-X and neo-Y, although male recombination does not occur at present in $D$.

483 albomicans (Satomura and Tamura 2016; see also Wei and Bachtrog 2019). A large number of shared

484 polymorphisms between neo-sex chromosomes was also reported in D. miranda (Nozawa et al. 2018),

485 which might also indicate male recombination in D. miranda and/or its recent ancestor. In D. americana,

486 available data are insufficient to count the number of shared polymorphisms between neo-X and neo-Y.

487 However, unlike those of D. miranda and D. pseudoobscura, and D. albomicans and D. nasuta, the

488 geographical distributions of $D$. americana and the closely-related species $D$. texana partly overlap

489 (Sillero et al. 2014). Therefore, if a hybrid male is backcrossed with a D. americana female, a neo-Y

490 pseudogene can be replaced with a functional gene that is highly expressed in the ovary, on the

491 orthologous autosome (i.e., chromosome 4) from D. texana. 


\section{Parallel pseudogenization of neo-Y-linked genes in D. miranda and D. albomicans}

This study reports a common evolutionary trajectory of the neo-sex chromosomes in Drosophila species. One of the most striking findings among these features may be the parallel pseudogenization of neo-Y-linked genes in D. miranda and D. albomicans. There is no enriched GO term in these genes with parallel pseudogenization, possibly due to a small number of such genes (only 35 of the 814 genes analyzed). However, a detailed inspection revealed that sexual conflict may be involved in at least some of the parallel pseudogenizations (Table S20). For example, Idgf6 is known to be involved in egg chamber tube morphogenesis (Zimmerman et al. 2017). In addition, JhI-26, which was originally identified as a juvenile hormone-inducible protein, was later reported as a sperm protein known to reduce male fertility under overexpression (Liu et al. 2014). Therefore, these genes may be under sexual conflict and thus may have been pseudogenized after neo-Y-linkage.

In conclusion, neo-sex chromosomes in Drosophila with independent origins share evolutionary required to evaluate whether the patterns reported in this study are applicable to other groups of

510 organisms.

\section{METHODS}

$513 \quad$ Flies

D. americana (strain 15010-0951.03, Millersberg, USA), D. texana (strain 15010-1041.00, St.

515 Francisville, USA), and D. novamexicana (strain 15010-1031.00, Grand Junction, USA) were obtained

516 from Drosophila Species Stock Center (https://www.drosophilaspecies.com/). D. albomicans (NG-3,

517 Nago, Japan), D. nasuta (strain G-86, Curepipe, Mauritius), and D. kohkoa (strain X-145, Kuala Lumpur,

518 Malaysia) have been maintained as living stocks at Tokyo Metropolitan University since the late 1970s. 


\section{Genome and RNA sequencing}

For genome sequencing, high molecular weight genomic DNA was extracted from males and

522 females separately. Whole genome shotgun sequencing was performed using the Illumina HiSeq 2500

523 and PacBio RSII/Sequel platforms (Pacific Biosciences, Menlo Park). PacBio long reads were used to

524 assemble the genome using the Hierarchical Genome Assembly Process (HGAP) 3 or HGAP4 (Chin et al.

525 2013). Illumina short-reads were then used to correct sequence errors using BWA (Li and Durbin 2009).

526 To obtain the neo-Y assemblies of D. americana and D. albomicans, we replaced the neo-X sequences

527 with male-specific variants. The genome assemblies of the nine species used in this study were evaluated

528 using BUSCO ver. 4.0.2 software (Simao et al. 2015).

For RNA sequencing, RNA was extracted from third instar larvae, pupae, adults, ovaries, and testes, and a cDNA library from each sample was sequenced using HiSeq 4000 or HiSeq X (Illumina, San Diego). We prepared two biological replicates for each condition to account for the fluctuation among

532 samples. Transcriptome assembly for each species was obtained using HISAT2-2.1.0 (Kim et al. 2019)

533 and StringTie 1.3.6 (Pertea et al. 2015). TransDecoder-5.5.0 (Haas et al. 2013) and Augustus-3.3.2

534 (Stanke and Waack 2003) were used to annotate coding sequences of expressed and non-expressed genes, 535 respectively. The expression level for each gene was estimated using the STAR 2.7 (Dobin et al. 2013)-

536 RSEM v1.3.1 (Li and Dewey 2011) pipeline.

\section{Gene classification}

To identify orthologs in the three closely-related species (i.e., D. miranda-D. pseudoobscura-D.

obscura, D. albomicans-D. nasuta-D. kohkoa, or D. americana-D. texana-D. novamexicana) and

541 inparalogs for each species, we conducted BLASTP (ver. 2.9.0+) search (Altschul et al. 1997). All

542 identified genes were classified into functional, silenced, disrupted, silenced-and-disrupted, and

543 unclassified genes based on coding sequence integrity and expression level. 
See SUPPLEMENTARY METHODS for complete methods with detailed descriptions.

\section{DATA ACCESS}

All sequence data reported in this study are available in the DDBJ Sequence Read Archive under the accession numbers DRA007619-26, DRA008052, DRA006582, and DRA004735 (see also Tables S1

550 and S4 for accession numbers for each run). Genome assemblies for the six Drosophila species are also

551 available in the DDBJ under the accession numbers BJEI01000001-BJEI01000834 for D. albomicans,

552 BJEH01000001-BJEH01000604 for D. nasuta, BJEL01000001-BJEL01000087 for D. kohkoa,

553 BJEJ01000001-BJEJ01000435 for D. americana, BJEK01000001-BJEK01000199 for D. texana, and

554 BJEM01000001-BJEM01000270 for D. novamexicana (see also Table S2). Other data are also available 555 upon a request to M.N.

\section{ACKNOWLEDGMENTS}

We thank Yasuko Urabe for her careful experiments. We also thank Kentaro M. and Tanaka, Ryo

559 Yamaguchi for their comments on earlier versions of our manuscripts. We would like to thank the staff of

560 Comparative Genomics Laboratory at National Institute of Genetics for supporting genome sequencing.

561 This work was supported by JSPS KAKENHI Grant Numbers 25711023, 15K14585, 17H05015,

562 221S0002, and 16H06279 and by the NBRP Genome Information Upgrading Program.

\section{DISCLOSURE DECLARATION}

The authors declare no conflicts of interest associated with this manuscript.

\section{AUTHOR CONTRIBUTIONS}

MN designed the research. MN, KS, SK, and KT prepared the fly materials. MN, YM, and AT

569 conducted DNA sequencing. MN conducted other experiments. MN analyzed the data. MN wrote the

570 draft version of the manuscript. All authors checked the manuscript and approved the research contents. 


\section{REFERENCES}

573 Alekseyenko AA, Ho JW, Peng S, Gelbart M, Tolstorukov MY, Plachetka A, Kharchenko PV, Jung YL, Gorchakov AA, Larschan E et al. 2012. Sequence-specific targeting of dosage compensation in Drosophila favors an active chromatin context. PLoS Genet 8: e1002646.

Bachtrog D, Mahajan S, Bracewell R. 2019. Massive gene amplification on a recently formed Drosophila Y chromosome. Nat Ecol Evol 3: 1587-1597.

Bachtrog D, Mank JE, Peichel CL, Kirkpatrick M, Otto SP, Ashman TL, Hahn MW, Kitano J, Mayrose I, Ming R et al. 2014. Sex determination: why so many ways of doing it? PLoS Biol 12: e1001899.

Bracewell R, Bachtrog D. 2020. Complex Evolutionary History of the Y Chromosome in Flies of the Drosophila obscura Species Group. Genome Biol Evol 12: 494-505.

Bracewell R, Chatla K, Nalley MJ, Bachtrog D. 2019. Dynamic turnover of centromeres drives karyotype evolution in Drosophila. Elife 8: e49002.

Celniker SE, Wheeler DA, Kronmiller B, Carlson JW, Halpern A, Patel S, Adams M, Champe M, Dugan SP, Frise E et al. 2002. Finishing a whole-genome shotgun: release 3 of the Drosophila melanogaster euchromatic genome sequence. Genome Biol 3: RESEARCH0079.

Charlesworth B. 2012. The role of background selection in shaping patterns of molecular evolution and variation: evidence from variability on the Drosophila X chromosome. Genetics 191: 233-246.

594 Chin CS, Alexander DH, Marks P, Klammer AA, Drake J, Heiner C, Clum A, Copeland A, Huddleston J, Eichler EE et al. 2013. Nonhybrid, finished microbial genome assemblies from long-read SMRT sequencing data. Nat Methods 10: 563-569. 
597

598

599

600

601

602

603

604

605

606

607

608

609

610

611

612

613

614

615

616

617

618

619

620

Cortez D, Marin R, Toledo-Flores D, Froidevaux L, Liechti A, Waters PD, Grutzner F, Kaessmann H. 2014. Origins and functional evolution of Y chromosomes across mammals. Nature 508: 488493.

Disteche CM. 2012. Dosage compensation of the sex chromosomes. Annu Rev Genet 46: 537-560.

Dobin A, Davis CA, Schlesinger F, Drenkow J, Zaleski C, Jha S, Batut P, Chaisson M, Gingeras TR. 2013. STAR: ultrafast universal RNA-seq aligner. Bioinformatics 29: 15-21.

Dupim EG, Goldstein G, Vanderlinde T, Vaz SC, Krsticevic F, Bastos A, Pinhao T, Torres M, David JR, Vilela CR et al. 2018. An investigation of Y chromosome incorporations in 400 species of Drosophila and related genera. PLoS Genet 14: e1007770.

Ellegren H, Hultin-Rosenberg L, Brunstrom B, Dencker L, Kultima K, Scholz B. 2007. Faced with inequality: chicken do not have a general dosage compensation of sex-linked genes. BMC Biol 5: 40.

Ellison C, Bachtrog D. 2019. Contingency in the convergent evolution of a regulatory network: Dosage compensation in Drosophila. PLoS Biol 17: e3000094.

Ellison CE, Bachtrog D. 2013. Dosage compensation via transposable element mediated rewiring of a regulatory network. Science 342: 846-850.

Flores SV, Evans AL, McAllister BF. 2008. Independent origins of new sex-linked chromosomes in the melanica and robusta species groups of Drosophila. BMC Evol Biol 8: 33.

Gartler SM. 2014. A brief history of dosage compensation. J Genet 93: 591-595.

Graves JA. 2016. Evolution of vertebrate sex chromosomes and dosage compensation. Nat Rev Genet 17: $33-46$.

Haas BJ, Papanicolaou A, Yassour M, Grabherr M, Blood PD, Bowden J, Couger MB, Eccles D, Li B, Lieber M et al. 2013. De novo transcript sequence reconstruction from RNA-seq using the Trinity platform for reference generation and analysis. Nat Protoc 8: 1494-1512. 
621

622

623

624

625

626

627

628

629

630

631

632

633

634

635

636

637

638

639

640

641

642

643

644

645

Hayakawa T, Suzuki-Hashido N, Matsui A, Go Y. 2014. Frequent expansions of the bitter taste receptor gene repertoire during evolution of mammals in the Euarchontoglires clade. Mol Biol Evol 31: 2018-2031.

John A, Vinayan K, Varghese J. 2016. Achiasmy: male fruit flies are not ready to mix. Front Cell Dev Biol 4: 75.

Kaiser VB, Zhou Q, Bachtrog D. 2011. Nonrandom gene loss from the Drosophila miranda neo-Y chromosome. Genome Biol Evol 3: 1329-1337.

Kim D, Paggi JM, Park C, Bennett C, Salzberg SL. 2019. Graph-based genome alignment and genotyping with HISAT2 and HISAT-genotype. Nat Biotechnol 37: 907-915.

Koerich LB, Wang X, Clark AG, Carvalho AB. 2008. Low conservation of gene content in the Drosophila Y chromosome. Nature 456: 949-951.

Li B, Dewey CN. 2011. RSEM: accurate transcript quantification from RNA-Seq data with or without a reference genome. BMC Bioinformatics 12: 323.

Li H, Durbin R. 2009. Fast and accurate short read alignment with Burrows-Wheeler transform. Bioinformatics 25: 1754-1760.

Lin F, Xing K, Zhang J, He X. 2012. Expression reduction in mammalian X chromosome evolution refutes Ohno's hypothesis of dosage compensation. Proc Natl Acad Sci USA 109: 11752-11757.

Liu C, Wang JL, Zheng Y, Xiong EJ, Li JJ, Yuan LL, Yu XQ, Wang YF. 2014. Wolbachia-induced paternal defect in Drosophila is likely by interaction with the juvenile hormone pathway. Insect Biochem Mol Biol 49: 49-58.

Mahajan S, Wei KH, Nalley MJ, Gibilisco L, Bachtrog D. 2018. De novo assembly of a young Drosophila Y chromosome using single-molecule sequencing and chromatin conformation capture. PLoS Biol 16: e2006348.

Mai D, Nalley MJ, Bachtrog D. 2020. Patterns of genomic differentiation in the Drosophila nasuta species complex. Mol Biol Evol 37: 208-220. 
646

647

648

649

650

651

652

653

654

655

656

657

658

659

660

661

662

663

664

665

666

667

668

669

670

McElroy KA, Kang H, Kuroda MI. 2014. Are we there yet? Initial targeting of the Male-Specific Lethal and Polycomb group chromatin complexes in Drosophila. Open Biol 4: 140006.

Meisel RP. 2020. Evolution of sex determination and sex chromosomes: a novel alternative paradigm. Bioessays 42: e1900212.

Muller HJ. 1932. Further studies on the nature and causes of gene mutations. Proceedings of the Sixth International Congress of Genetics213-255.

Nei M, Niimura Y, Nozawa M. 2008. The evolution of animal chemosensory receptor gene repertoires: roles of chance and necessity. Nat Rev Genet 9: 951-963.

Niimura Y, Nei M. 2007. Extensive gains and losses of olfactory receptor genes in mammalian evolution. PLoS One 2: e708.

Nozawa M, Fukuda N, Ikeo K, Gojobori T. 2014. Tissue- and stage-dependent dosage compensation on the neo-X chromosome in Drosophila pseudoobscura. Mol Biol Evol 31: 614-624.

Nozawa M, Ikeo K, Gojobori T. 2018. Gene-by-gene or localized dosage compensation on the neo-X chromosome in Drosophila miranda. Genome Biol Evol 10: 1875-1881.

Nozawa M, Nei M. 2007. Evolutionary dynamics of olfactory receptor genes in Drosophila species. Proc Natl Acad Sci USA 104: 7122-7127.

Nozawa M, Onizuka K, Fujimi M, Ikeo K, Gojobori T. 2016. Accelerated pseudogenization on the neo-X chromosome in Drosophila miranda. Nat Commun 7: 13659.

Ohno S. 1967. Sex chromosomes and sex-linked genes. Springer-Verlag.

Pertea M, Pertea GM, Antonescu CM, Chang TC, Mendell JT, Salzberg SL. 2015. StringTie enables improved reconstruction of a transcriptome from RNA-seq reads. Nat Biotechnol 33: 290-295.

Satomura K, Tamura K. 2016. Ancient Male Recombination Shaped Genetic Diversity of Neo-Y Chromosome in Drosophila albomicans. Mol Biol Evol 33: 367-374.

Seppey M, Manni M, Zdobnov EM. 2019. BUSCO: assessing genome assembly and annotation completeness. Methods Mol Biol 1962: 227-245. 
671 Sillero N, Reis M, Vieira CP, Vieira J, Morales-Hojas R. 2014. Niche evolution and thermal adaptation in the temperate species Drosophila americana. J Evol Biol 27: 1549-1561.

673 Simao FA, Waterhouse RM, Ioannidis P, Kriventseva EV, Zdobnov EM. 2015. BUSCO: assessing genome assembly and annotation completeness with single-copy orthologs. Bioinformatics 31:

675

676

677

678

679

680

681

682

683

684

685

686

687

688

689

690

691

692

693

694

695

696 3210-3212.

Stanke M, Waack S. 2003. Gene prediction with a hidden Markov model and a new intron submodel. Bioinformatics 19 Suppl 2: ii215-225.

Valsecchi CIK, Basilicata MF, Georgiev P, Gaub A, Seyfferth J, Kulkarni T, Panhale A, Semplicio G, Manjunath V, Holz $\mathrm{H}$ et al. 2021. RNA nucleation by MSL2 induces selective X chromosome compartmentalization. Nature 589: 137-142.

Vicoso B, Bachtrog D. 2011. Lack of global dosage compensation in Schistosoma mansoni, a femaleheterogametic parasite. Genome Biol Evol 3: 230-235.

-. 2015. Numerous transitions of sex chromosomes in Diptera. PLoS Biol 13: e1002078.

Vieira CP, Coelho PA, Vieira J. 2003. Inferences on the evolutionary history of the Drosophila americana polymorphic X/4 fusion from patterns of polymorphism at the X-linked paralytic and elav genes. Genetics 164: 1459-1469.

Wei KH, Bachtrog D. 2019. Ancestral male recombination in Drosophila albomicans produced geographically restricted neo-Y chromosome haplotypes varying in age and onset of decay. PLoS Genet 15: e1008502.

Yanai I, Benjamin H, Shmoish M, Chalifa-Caspi V, Shklar M, Ophir R, Bar-Even A, Horn-Saban S, Safran M, Domany E et al. 2005. Genome-wide midrange transcription profiles reveal expression level relationships in human tissue specification. Bioinformatics 21: 650-659.

Zha X, Xia Q, Duan J, Wang C, He N, Xiang Z. 2009. Dosage analysis of Z chromosome genes using microarray in silkworm, Bombyx mori. Insect Biochem Mol Biol 39: 315-321.

Zhou Q, Bachtrog D. 2015. Ancestral chromatin configuration constrains chromatin evolution on differentiating sex chromosomes in Drosophila. PLoS Genet 11: e1005331. 
697 Zhou Q, Ellison CE, Kaiser VB, Alekseyenko AA, Gorchakov AA, Bachtrog D. 2013. The epigenome of

698 evolving Drosophila neo-sex chromosomes: dosage compensation and heterochromatin

699 formation. PLoS Biol 11: e1001711.

700 Zhou Q, Zhang J, Bachtrog D, An N, Huang Q, Jarvis ED, Gilbert MT, Zhang G. 2014. Complex

701 evolutionary trajectories of sex chromosomes across bird taxa. Science 346: 1246338.

702 Zimmerman SG, Merrihew GE, MacCoss MJ, Berg CA. 2017. Proteomics analysis identifies orthologs of 703 human chitinase-like proteins as inducers of tube morphogenesis defects in Drosophila melanogaster. Genetics 206: 973-984. 